\section{Diffusion weighted MR imaging}

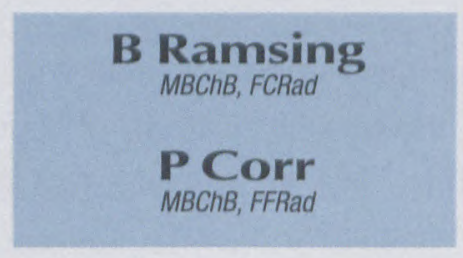

lower concentration along a gradient is called diffusion. However, even when the ink has distributed itself throughout the water and there are no concentration gradients, there are still random movements of ink molecules. This intrinsic kinetic energy of molecules is called Brownian motion. Water within tissues displays Brownian motion. The diffusion process is three dimensional and is distributed in all directions perpendicular to its origin. This is called isotropic distribution. However, in tissues water molecule movement is determined by the local molecular environment and cannot distribute itself in an isotropic manner. This distribution is called anisotropy. An example is water molecules within neurones, in which the molecules move parallel to the axons rather than at right angles to them. Because of anisotropy, a three dimensional data acquisition is performed during imaging.

Using conventional spin echo imaging, diffusion causes minimal signal reduction, but with very strong gradients it is possible to obtain diffusion weighted images (DWI). DWI is very sensitive to motion artefacts such as breathing and cardiac pulsation. Fast imaging using echo planar techniques will reduce motion artefacts and improve image quality. The images have a poor signal-to-noise ratio compared to conventional spin echo sequences of the brain, with susceptibility artefacts adjacent to the skull and sinuses. Regions of high diffusion have a low intensity and appear dark on the scan, while regions of low diffusion have a relatively increased intensity and appear bright on the scan. The apparent diffusion coefficient (ADC) is a quantitative 


\section{Diffusion weighted MR imaging}

\section{from page 4}

measure of the degree of diffusion in a tissue or organ. The average value for the brain is $2100 \times 10^{6} \mathrm{~mm}^{2} / \mathrm{s}$. The other important concept is the $b$ value. This is a measure of the strength and duration of the MR gradient's pulses, as well as the time between gradient pulses and the gyromagnetic ratio. The higher the $b$ value, the better the differentiation between tissues of different diffusion coefficients and hence lesion conspicuity. DWI is best between a $b$ value of 600 to 1000 $\mathrm{mm}^{2} / \mathrm{sec}^{1}$

DWI is essentially a cellular energy test. DWI indicates cell osmolality which is dependent on the integrity of the cell membrane pump and cell energy levels of ATP. With depletion of cell energy supplies of ATP, the cellular pump fails and the cell swells resulting in cytotoxic oedema. This results in a marked decrease in diffusion (ADC) and a focal bright signal on the scan.

\section{Clinical applications}

\section{Acute stroke}

With acute stroke, cytotoxic oedema develops in the neurones and the diffusion of water molecules decreases. This is thought to be due to decreased Na K-ATPase enzyme activity in the cell membrane, and therefore decreased water transport. Water becomes trapped within the cell. Water viscosity is increased due to the dissociation of large macromolecules and the cellular fluid becomes a gel. This also decreases water diffusion. In animal models, the decreased diffusion is detected as a high intensity focal lesion within 45 minutes of experimental vascular occlusion. ${ }^{2}$ After two days, diffusion returns to normal and then increases. In humans DWI detects changes as early as $38 \mathrm{~min}$ utes and diffusion decreases over 24 hours to 4 days (Figure 1). Diffusion

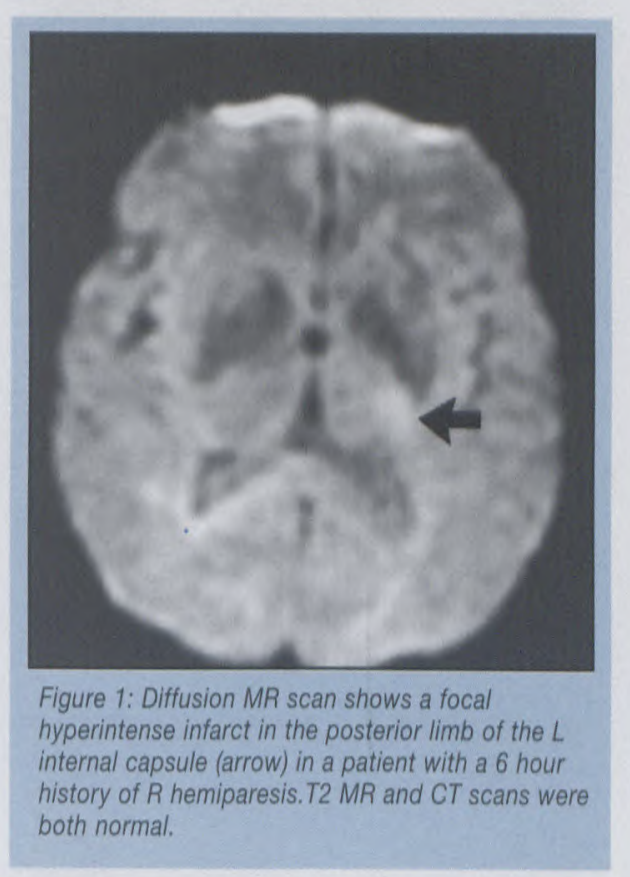

returns to normal at 5 to 10 days post ictus. The slow return to normal diffusion levels is due to persistent cytotoxic oedema as well as vasogenic oedema. This is a consequence of cell membrane disruption and resultant extracellular oedema which increases diffusion levels. Reversibility of diffusion to normal levels can be consistently detected in the animal model, with a threshold ADC above which no permanent tissue injury is detected. In humans this has yet to be achieved. ${ }^{3}$

CT and MR cannot detect cerebral infarction before 8 hours when thrombolytic therapy is most effective; however DWI is able to provide this information. ${ }^{4} \mathrm{~A}$ lesion with decreased diffusion correlates strongly with cytotoxic oedema and infarction. The absence of decreased diffusion correlates with the absence of infarction, and rather suggests stroke mimics such as transient ischaemic attacks, migraine, and metabolic causes.

\section{Subacute stroke}

DWI is useful in differentiating white matter infarcts from the T2 hyperintensities associated with aging. On DWI, infarcts are hyperintense while the aging changes demonstrate no diffusion change. DWI is also useful to distinguish subacute from chronic infarction, which is impossible with spin echo T2 imaging. Subacute infarcts are hyperintense on DWI due to decreased diffusion from cytotoxic oedema, while chronic infarcts are hypointense as a result of increased diffusion from vasogenic oedema and increased extracellular water. ${ }^{5} \mathrm{~T}{ }^{*}$ susceptibility effects are more readily detected with DWI as opposed to spin echo imaging, so that haemorrhagic infarction is more easily identified.

\section{Neonatal ischaemia}

DWI is useful in imaging hypoxic ischaemic encephalopathy in neonates. The white matter is unmyelinated and CT and MR detection of infarction are difficult. DWI easily detects infarcted brain where diffusion is decreased compared to normal brain. DWI can differentiate between embolic infarcts and cortical laminar necrosis. This is important clinically as neonates with embolic infarcts have a better prognosis. ${ }^{5}$

\section{Venous infarct}

DWI distinguishes between venous infarction and extracellular oedema resulting from occluded cortical veins and venous sinuses. Infarction is hyperintense while the surrounding extracellular vasogenic oedema is hypointense. 
from page 5

\section{Stroke mimics}

DWI can detect disorders that may mimic acute stroke or cerebral infarction. Included in this group are atypical migraine and transient ischaemic attacks (TIA). In these patients diffusion is normal and infarction can be excluded.

There are also disorders with both vasogenic oedema and sometimes cytotoxic oedema that can mimic stroke. ${ }^{5}$ Only DWI can distinguish between cytotoxic and vasogenic oedema. This group includes: eclampsia, hypertensive encephalopathy, posterior leucoencephalopathy and hyperperfusion injury (Figures 2a,b,c).

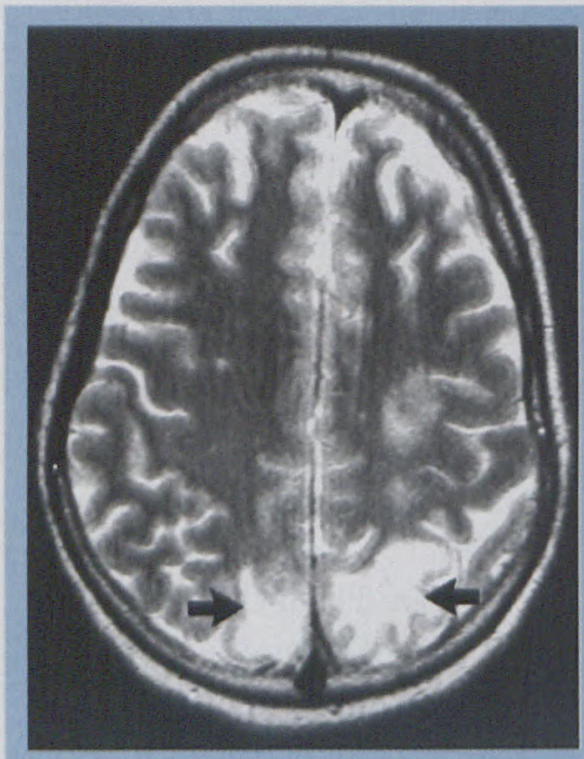

Figure 2a: Patient with eclampsia. T2 MR demonstrates hyperintense lesions in both occipital poles representing focal oedema (arrows).

Vasogenic oedema is characterized by increased diffusion of water. This is due to increased extracellular water and increased membrane permeability to water.

\section{Neoplasms}

DWI is most useful in characterizing extra-axial tumours, especially differentiating arachnoid cysts from epidermoids. ${ }^{6}$ Both have low intensity

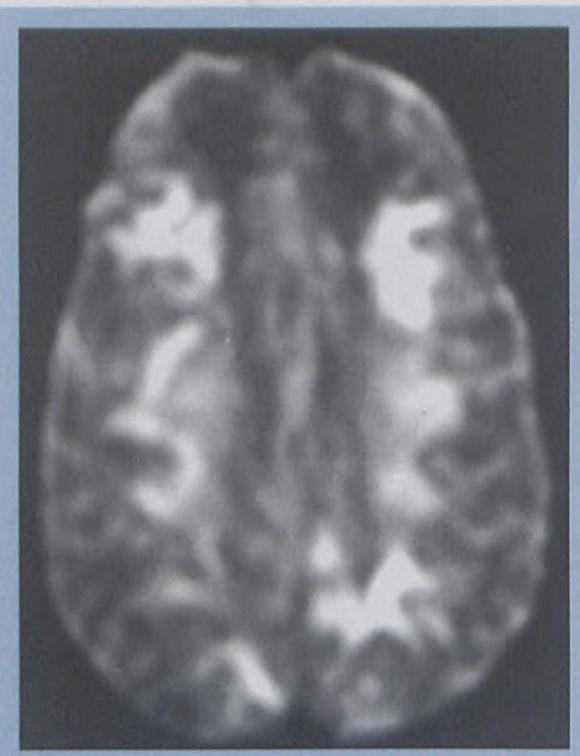

Figure 2b: Diffusion MR demonstrates extensive vasogenic oedema (arrow).

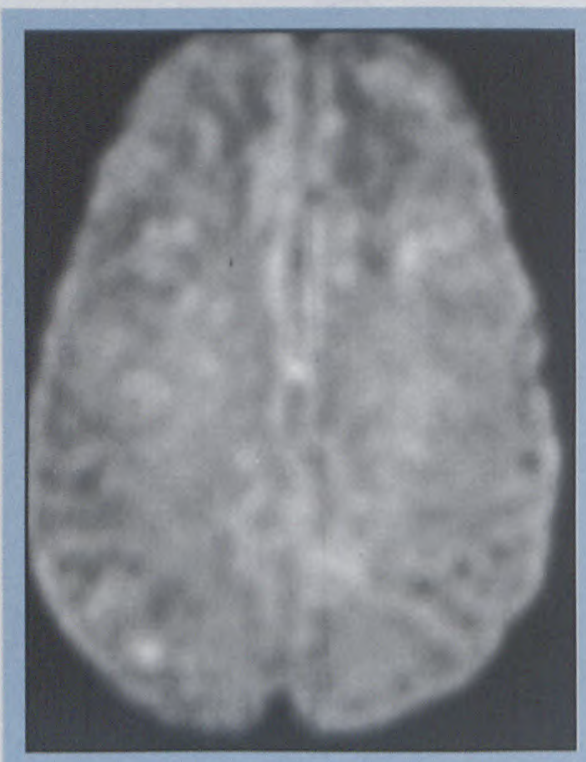

Figure 2c: Follow-up diffusion scan at 48 hours after start of treatment confirms resolved vasogenic oedema.

on $\mathrm{Tl}$ and high intensity on $\mathrm{T} 2$. However, epidermoids have decreased diffusion and appear hyperintense on DWI; arachnoid cysts have increased diffusion and appear hypointense on DWI. Intra-axial tumour morphology has been investigated using DWI. DWI can distinguish between solid and cystic components of tumours as well as tumour and surrounding vasogenic oedema.

\section{Trauma and haemorrhage}

DWI may have an important role in the detection of ischaemia related to cerebral trauma. ${ }^{8}$ Trauma causes increased cortical diffusion due to increased extracellular water. However, with severe head injury, ischaemia due to decreased cerebral blood flow can be detected as cytotoxic oedema and as hyperintense parenchymal lesions on DWI. DWI is very sensitive to parenchymal haemorrhage due to $\mathrm{T} 2 *$ susceptibility effects.

\section{Infection}

Pyogenic infection, such as abscesses, have decreased diffusion and appear as hyperintense lesions on DWI. ${ }^{5}$ Other ring enhancing lesions such as tumours have increased diffusion and thus appear hypointense. This allows characterization of inflammatory masses. Empyemas have very low diffusion and appear hyperintense compared to extraxial hygromas which have increased diffusion and appear hypointense on DWI.

\section{Spine}

DWI of the spine successfully differentiates benign osteoporotic from malignant fractures. Osteoporotic fractures have hypointense bone marrow signal, while metastases are hyperintense to the normal bone marrow signal. ${ }^{9}$

\section{Conclusion}

DWI is an important new MR technique which allows investigation of tissues at a molecular level. It has enhanced our understanding of the pathophysiology of stroke and will probably become the most important imaging modality in early detection of infarction. DWI shows promise in 